\title{
WHEN LATTICE HOMOMORPHISMS OF ARCHIMEDEAN VECTOR LATTICES ARE RIESZ HOMOMORPHISMS
}

\author{
MOHAMED ALI TOUMI
}

\author{
(Received 4 March 2008; accepted 11 September 2008) \\ Communicated by A. J. Pryde
}

\begin{abstract}
Let $A, B$ be Archimedean vector lattices and let $\left(u_{i}\right)_{i \in I},\left(v_{i}\right)_{i \in I}$ be maximal orthogonal systems of $A$ and $B$, respectively. In this paper, we prove that if $T$ is a lattice homomorphism from $A$ into $B$ such that $T\left(\lambda u_{i}\right)=\lambda v_{i}$ for each $\lambda \in \mathbb{R}_{+}$and $i \in I$, then $T$ is linear. This generalizes earlier results of Ercan and Wickstead (Math. Nachr 279 (9-10) (2006), 1024-1027), Lochan and Strauss (J. London Math. Soc. (2) 25 (1982), 379-384), Mena and Roth (Proc. Amer. Math. Soc. 71 (1978), 11-12) and Thanh (Ann. Univ. Sci. Budapest. Eotvos Sect. Math. 34 (1992), 167-171).
\end{abstract}

2000 Mathematics subject classification: primary 46A40; secondary 47B65.

Keywords and phrases: weak order unit, lattice homomorphism, Riesz homomorphism.

\section{Introduction}

In this paper, we give some conditions under which a lattice homomorphism is linear. Our starting point is a theorem of Mena and Roth [6] (generalized by Thanh [8], by Lochan and Strauss [4] and recently by Ercan and Wickstead [3]) where $T$ is a lattice homomorphism of $C(X)$-spaces and hence via the Kakutani representation theorem, $T$ acts between two uniformly complete Archimedean vector lattices $A$ and $B$ with (strong or weak) order units. Perhaps the most general result in this direction is the work of Ercan and Wickstead [3]. More precisely, they deduced from the theorem of Mena and Roth and by using the Kakutani representation theorem, that if $A$ and $B$ are uniformly complete Archimedean vector lattices $A$ and $B$ with weak order units $e_{1} \in A$ and $e_{2} \in B$ and if $T$ is a lattice homomorphism from $A$ to $B$, such that $T\left(\lambda e_{1}\right)=\lambda e_{2}$ for each $\lambda \in \mathbb{R}$, then $T$ is linear. Finally, using the same argument, they gave a corresponding result for the case where $T$ acts between two uniformly complete Archimedean vector lattices with disjoint complete systems of projection, for example, between two $\sigma$-Dedekind complete vector lattices. To the best of our knowledge, there

(C) 2009 Australian Mathematical Publishing Association Inc. 1446-7887/2009 \$16.00 
is no proof, without use of representation theory, of these versions of the Mena-Roth theorem. The first aim of this paper is to give not only a proof of the theorem of Mena and Roth, which relies on a new, constructive and intrinsic approach but also it does not make use of the uniformly completeness of $A$ and $B$. Finally, we are concerned with lattice homomorphisms that act between Archimedean vector lattices. More precisely, we prove that if $A, B$ are Archimedean vector lattices, if $\left(u_{i}\right)_{i \in I},\left(v_{i}\right)_{i \in I}$ are maximal orthogonal systems of $A$ and $B$, respectively, and if $T$ is a lattice homomorphism from $A$ into $B$ such that $T\left(\lambda u_{i}\right)=\lambda v_{i}$ for each $\lambda \in \mathbb{R}_{+}$and $i \in I$, then $T$ is linear. This generalizes earlier results of $[3,4,6]$ and [8].

We take it for granted that the reader is familiar with the notions of vector lattices (or Riesz spaces) and operators between them. For terminology, notation and concepts that are not explained in this paper we refer to the standard monographs $[1,5,7]$.

\section{Definitions and notations}

We assume throughout this paper that all vector lattices (or Riesz spaces) under consideration are Archimedean.

A map $T$ between vector lattices $A$ and $B$ is called a lattice homomorphism if

$$
T(a \wedge b)=T(a) \wedge T(b) \quad \text { and } \quad T(a \vee b)=T(a) \vee T(b) \quad \text { for each } a, b \in A .
$$

A linear lattice homomorphism is called Riesz homomorphism.

Let $A$ be a (real) vector lattice. A vector subspace $I$ of $A$ is called an order ideal (or $o$-ideal) whenever $|a| \leq|b|$ and $b \in I$ imply $a \in I$. Every $o$-ideal is a vector sublattice of $A$. The principal $o$-ideal generated by $0 \leq e \in A$ is denoted by $A_{e}$ and it is a sublattice of $A$.

Let $A$ be a vector lattice, let $0 \leq v \in A$, the sequence $\left\{a_{n}, n=1,2, \ldots\right\}$ in $A$ is called $(v)$ relatively uniformly convergent to $a \in A$ if, for every real number $\varepsilon>0$, there exists a natural number $n_{\varepsilon}$ such that $\left|a_{n}-a\right| \leq \varepsilon v$ for all $n \geq n_{\varepsilon}$. This will be denoted by $a_{n} \rightarrow a(v)$. If $a_{n} \rightarrow a(v)$ for some $0 \leq v \in A$, then the sequence $\left\{a_{n}, n=1,2, \ldots\right\}$ is called (relatively) uniformly convergent to $a$, which is denoted by $a_{n} \rightarrow a(r . u$.). The notion of $(v)$ relatively uniformly Cauchy sequence is defined in the obvious way. Relatively uniformly limits are unique in Archimedean vector lattices, see [5, Theorem 63.2]. A vector lattice $A$ is called relatively uniformly complete whenever every relatively uniformly Cauchy sequence in $A$ has a unique limit. Every relatively uniformly complete vector lattice is Archimedean. Let $A$ be a vector lattice (or Riesz space). A subset $S$ of the positive cone $A^{+}$is called an orthogonal system of $A$ if $0 \notin S$ and if $u \wedge v=0$ for each pair $(u, v)$ of distinct elements of $S$. It is clear from Zorn's lemma that every orthogonal system of $A$ is contained in a maximal orthogonal system. An element $e$ of a vector lattice $A$ is called weak order unit (strong order unit) of $A$ whenever $\{e\}$ is a maximal orthogonal system of $A$ (respectively, $A_{e}=A$ ). An $\ell$-algebra $A$ is called an $f$-algebra if $A$ verifies the property that $a \wedge b=0$ and $c \geq 0$ imply $a c \wedge b=c a \wedge b=0$. Any $f$-algebra is automatically commutative and has positive squares. 


\section{The main results}

Our main goal is to establish the result corresponding to the Mena-Roth theorem for lattice homomorphisms on vector lattice with (strong and weak) order units. The following proposition is an essential ingredient for our main results.

Before continuing with the next result, we recall the following notion.

Let $A$ be a vector lattice and let $0 \leq a \in A$. An element $0 \leq e \in A$ is called $a$ component of $a$ if $e \wedge(a-e)=0$.

PROPOSITION 1. Let A be a Dedekind complete vector lattice with strong order unit $e$, let $e_{1}, e_{2}, \ldots, e_{n}$ be components of $e$ and let $B$ be a vector lattice. If $T$ is a lattice homomorphism from $A$ into $B$ such that $T(\lambda e)=\lambda T(e)$ for each $\lambda \in \mathbb{R}_{+}$, then $T$ satisfies the following property:

$$
T\left(\sum_{i=1}^{n} \lambda_{i} e_{i}\right)=\sum_{i=1}^{n} \lambda_{i} T\left(e_{i}\right) \quad \forall \lambda_{i} \in \mathbb{R}_{+}(1 \leq i \leq n) .
$$

PRoof. Step 1. We show first that $T\left(\lambda e_{1}\right)=\lambda T\left(e_{1}\right)$ for each $\lambda \in \mathbb{R}_{+}$. Let us denote $e_{i}^{c}=e-e_{i}$, for all $1 \leq i \leq n$. Since $e_{1}^{c}+e_{1}=e_{1}^{c} \vee e_{1}=e, e_{1}^{c} \wedge e_{1}=0$ and since $T$ is a lattice homomorphism, then $T\left(e_{1}^{c}\right) \wedge T\left(e_{1}\right)=0$. It follows that

$$
\begin{aligned}
T(\lambda e) & =T\left(\lambda\left(e_{1}^{c} \vee e_{1}\right)\right) \\
& =T\left(\lambda e_{1}^{c} \vee \lambda e_{1}\right) \\
& =T\left(\lambda e_{1}^{c}\right) \vee T\left(\lambda e_{1}\right) \\
& =T\left(\lambda e_{1}^{c}\right)+T\left(\lambda e_{1}\right) \quad \forall \lambda \in \mathbb{R}_{+} .
\end{aligned}
$$

By the fact that

$$
\begin{aligned}
T(\lambda e) & =\lambda T(e) \\
& =\lambda T\left(e_{1}^{c}+e_{1}\right) \\
& =\lambda T\left(e_{1}^{c} \vee e_{1}\right) \\
& =\lambda T\left(e_{1}^{c}\right) \vee \lambda T\left(e_{1}\right) \quad \\
& =\lambda T\left(e_{1}^{c}\right)+\lambda T\left(e_{1}\right) \quad \forall \lambda \in \mathbb{R}_{+},
\end{aligned}
$$

we deduce that

$$
T\left(\lambda e_{1}^{c}\right)-\lambda T\left(e_{1}^{c}\right)=\lambda T\left(e_{1}\right)-T\left(\lambda e_{1}\right) \quad \forall \lambda \in \mathbb{R}_{+} .
$$

Moreover, since $\lambda e_{1}^{c} \wedge e_{1}=e_{1}^{c} \wedge \lambda e_{1}=0$, for each $\lambda \in \mathbb{R}_{+}$and since $T$ is a lattice homomorphism, we have

$$
T\left(\lambda e_{1}^{c}\right) \wedge T\left(e_{1}\right)=\lambda T\left(e_{1}^{c}\right) \wedge T\left(e_{1}\right)=0 \quad \forall \lambda \in \mathbb{R}_{+} .
$$

Hence,

$$
\left|T\left(\lambda e_{1}^{c}\right)-\lambda T\left(e_{1}^{c}\right)\right| \wedge \lambda T\left(e_{1}\right)=0 \quad \forall \lambda \in \mathbb{R}_{+} .
$$


Using the same argument, we have

$$
\left|T\left(\lambda e_{1}^{c}\right)-\lambda T\left(e_{1}^{c}\right)\right| \wedge T\left(\lambda e_{1}\right)=0 \quad \forall \lambda \in \mathbb{R}_{+} .
$$

Therefore

$$
\left|T\left(\lambda e_{1}^{c}\right)-\lambda T\left(e_{1}^{c}\right)\right| \wedge\left|\lambda T\left(e_{1}\right)-T\left(\lambda e_{1}\right)\right|=0 \quad \forall \lambda \in \mathbb{R}_{+} .
$$

Hence,

$$
T\left(\lambda e_{1}^{c}\right)-\lambda T\left(e_{1}^{c}\right)=\lambda T\left(e_{1}\right)-T\left(\lambda e_{1}\right)=0 \quad \forall \lambda \in \mathbb{R}_{+} .
$$

Step 2. We show that $T\left(\alpha e_{1}+\beta e_{2}\right)=\alpha T\left(e_{1}\right)+\beta T\left(e_{2}\right)$ for each $\alpha, \beta \in \mathbb{R}_{+}$. To this end, we remark that $A_{e}=A$ can be seen as an $f$-algebra with $e$ as unit (where its $f$-algebra multiplication is denoted by juxtaposition; see [2, Remark 19.5]). In order to reach our aim, we first show that $T\left(\lambda e_{1} e_{2}\right)=\lambda T\left(e_{1} e_{2}\right)$ and $T\left(\lambda e_{1}^{c} e_{2}^{c}\right)=\lambda T\left(e_{1}^{c} e_{2}^{c}\right)$ for each $\lambda \in \mathbb{R}_{+}$. To this end, let $\lambda \in \mathbb{R}_{+}$. Since

$$
\begin{aligned}
\lambda e & =\lambda e^{2} \\
& =\lambda\left(e_{1}^{c}+e_{1}\right)\left(e_{2}^{c}+e_{2}\right) \\
& =\lambda e_{1} e_{2}+\lambda e_{1} e_{2}^{c}+\lambda e_{1}^{c} e_{2}+\lambda e_{1}^{c} e_{2}^{c} .
\end{aligned}
$$

We point out that $\lambda e_{1} e_{2}, \lambda e_{1} e_{2}^{c}, \lambda e_{1}^{c} e_{2}$ and $\lambda e_{1}^{c} e_{2}^{c}$ are mutually disjoint. Then by using the fact that $T$ is a lattice homomorphism, we obtain

$$
\begin{aligned}
T(\lambda e) & =T\left(\lambda e_{1} e_{2}+\lambda e_{1} e_{2}^{c}+\lambda e_{1}^{c} e_{2}+\lambda e_{1}^{c} e_{2}^{c}\right) \\
& =T\left(\lambda e_{1} e_{2} \vee \lambda e_{1} e_{2}^{c} \vee \lambda e_{1}^{c} e_{2} \vee \lambda e_{1}^{c} e_{2}^{c}\right) \\
& =T\left(\lambda e_{1} e_{2}\right) \vee T\left(\lambda e_{1} e_{2}^{c}\right) \vee T\left(\lambda e_{1}^{c} e_{2}\right) \vee T\left(\lambda e_{1}^{c} e_{2}^{c}\right) \\
& =T\left(\lambda e_{1} e_{2}\right)+T\left(\lambda e_{1} e_{2}^{c}\right)+T\left(\lambda e_{1}^{c} e_{2}\right)+T\left(\lambda e_{1}^{c} e_{2}^{c}\right)
\end{aligned}
$$

As

$$
\begin{aligned}
T(\lambda e) & =\lambda T(e) \\
& =\lambda T\left(e_{1} e_{2}+e_{1} e_{2}^{c}+e_{1}^{c} e_{2}+e_{1}^{c} e_{2}^{c}\right) \\
& =\lambda T\left(e_{1} e_{2} \vee e_{1} e_{2}^{c} \vee e_{1}^{c} e_{2} \vee e_{1}^{c} e_{2}^{c}\right) \\
& =\lambda\left(T\left(e_{1} e_{2}\right) \vee T\left(e_{1} e_{2}^{c}\right) \vee T\left(e_{1}^{c} e_{2}\right) \vee T\left(e_{1}^{c} e_{2}^{c}\right)\right) \\
& =\lambda\left(T\left(e_{1} e_{2}\right)+T\left(e_{1} e_{2}^{c}\right)+T\left(e_{1}^{c} e_{2}\right)+T\left(e_{1}^{c} e_{2}^{c}\right)\right) \\
& =\lambda T\left(e_{1} e_{2}\right)+\lambda T\left(e_{1} e_{2}^{c}\right)+\lambda T\left(e_{1}^{c} e_{2}\right)+\lambda T\left(e_{1}^{c} e_{2}^{c}\right) .
\end{aligned}
$$

Thus,

$$
\begin{aligned}
T\left(\lambda e_{1} e_{2}\right)-\lambda T\left(e_{1} e_{2}\right)= & \lambda T\left(e_{1} e_{2}^{c}\right)+\lambda T\left(e_{1}^{c} e_{2}\right)+\lambda T\left(e_{1}^{c} e_{2}^{c}\right) \\
& -\left(T\left(\lambda e_{1} e_{2}^{c}\right)+T\left(\lambda e_{1}^{c} e_{2}\right)+T\left(\lambda e_{1}^{c} e_{2}^{c}\right)\right) .
\end{aligned}
$$


Let

$$
X=\left|T\left(\lambda e_{1} e_{2}\right)-\lambda T\left(e_{1} e_{2}\right)\right|
$$

and let

$$
Y=\left|\lambda T\left(e_{1} e_{2}^{c}\right)+\lambda T\left(e_{1}^{c} e_{2}\right)+\lambda T\left(e_{1}^{c} e_{2}^{c}\right)-\left(T\left(\lambda e_{1} e_{2}^{c}\right)+T\left(\lambda e_{1}^{c} e_{2}\right)+T\left(\lambda e_{1}^{c} e_{2}^{c}\right)\right)\right| .
$$

Since $\lambda e_{1} e_{2} \wedge \lambda e_{1} e_{2}^{c}=\lambda e_{1} e_{2} \wedge e_{1} e_{2}^{c}=0$ and since $T$ is a lattice homomorphism, it follows that

$$
T\left(\lambda e_{1} e_{2}\right) \wedge \lambda T\left(e_{1} e_{2}^{c}\right)=T\left(\lambda e_{1} e_{2}\right) \wedge T\left(\lambda e_{1} e_{2}^{c}\right)=0
$$

and

$$
\lambda T\left(e_{1} e_{2}\right) \wedge \lambda T\left(e_{1} e_{2}^{c}\right)=\lambda T\left(e_{1} e_{2}\right) \wedge T\left(\lambda e_{1} e_{2}^{c}\right)=0 .
$$

Hence,

$$
\left|\lambda T\left(e_{1} e_{2}^{c}\right)-T\left(\lambda e_{1} e_{2}^{c}\right)\right| \wedge T\left(\lambda e_{1} e_{2}\right)=0
$$

and

$$
\left|\lambda T\left(e_{1} e_{2}^{c}\right)-T\left(\lambda e_{1} e_{2}^{c}\right)\right| \wedge \lambda T\left(e_{1} e_{2}\right)=0 .
$$

Therefore,

$$
\left|\lambda T\left(e_{1} e_{2}^{c}\right)-T\left(\lambda e_{1} e_{2}^{c}\right)\right| \wedge X=0 .
$$

Using the same argument

$$
\left|\lambda T\left(e_{1}^{c} e_{2}\right)-T\left(\lambda e_{1}^{c} e_{2}\right)\right| \wedge X=0
$$

and

$$
\left|\lambda T\left(e_{1}^{c} e_{2}^{c}\right)-T\left(\lambda e_{1}^{c} e_{2}^{c}\right)\right| \wedge X=0 .
$$

As a conclusion $X \wedge Y=0$. It follows that

$$
\begin{aligned}
T\left(\lambda e_{1} e_{2}\right)-\lambda T\left(e_{1} e_{2}\right)= & \lambda T\left(e_{1} e_{2}^{c}\right)+\lambda T\left(e_{1}^{c} e_{2}\right)+\lambda T\left(e_{1}^{c} e_{2}^{c}\right) \\
& -\left(T\left(\lambda e_{1} e_{2}^{c}\right)+T\left(\lambda e_{1}^{c} e_{2}\right)+T\left(\lambda e_{1}^{c} e_{2}^{c}\right)\right) \\
= & 0 .
\end{aligned}
$$

By using the same argument, we obtain

$$
\begin{aligned}
& \lambda T\left(e_{1} e_{2}^{c}\right)=T\left(\lambda e_{1} e_{2}^{c}\right) \\
& \lambda T\left(e_{1}^{c} e_{2}\right)=T\left(\lambda e_{1}^{c} e_{2}\right) \\
& \lambda T\left(e_{1}^{c} e_{2}^{c}\right)=T\left(\lambda e_{1}^{c} e_{2}^{c}\right) .
\end{aligned}
$$

Now let $\alpha, \beta \in \mathbb{R}_{+}$. Since

$$
\begin{aligned}
\alpha e_{1}+\beta e_{2} & =\alpha e_{1} e+\beta e_{2} e \\
& =\alpha e_{1}\left(e_{2}^{c}+e_{2}\right)+\beta e_{2}\left(e_{1}^{c}+e_{1}\right) \\
& =\alpha e_{1} e_{2}^{c}+\alpha e_{1} e_{2}+\beta e_{2} e_{1}^{c}+\beta e_{2} e_{1} \\
& =(\alpha+\beta) e_{1} e_{2}+\alpha e_{1} e_{2}^{c}+\beta e_{2} e_{1}^{c}
\end{aligned}
$$


A simple combination between the fact that $(\alpha+\beta) e_{1} e_{2}, \alpha e_{1} e_{2}^{c}$ and $\beta e_{2} e_{1}^{c}$ are mutually disjoint and the fact that $T$ is a lattice homomorphism, we get by using the equalities (3.1), (3.2), (3.3) and (3.4)

$$
\begin{aligned}
T\left(\alpha e_{1}+\beta e_{2}\right) & =T\left((\alpha+\beta) e_{1} e_{2}+\alpha e_{1} e_{2}^{c}+\beta e_{2} e_{1}^{c}\right) \\
& =T\left((\alpha+\beta) e_{1} e_{2} \vee \alpha e_{1} e_{2}^{c} \vee \beta e_{2} e_{1}^{c}\right) \\
& =T\left((\alpha+\beta) e_{1} e_{2}\right) \vee T\left(\alpha e_{1} e_{2}^{c}\right) \vee T\left(\beta e_{2} e_{1}^{c}\right) \\
& =T\left((\alpha+\beta) e_{1} e_{2}\right)+T\left(\alpha e_{1} e_{2}^{c}\right)+T\left(\beta e_{2} e_{1}^{c}\right) \\
& =(\alpha+\beta) T\left(e_{1} e_{2}\right)+\alpha T\left(e_{1} e_{2}^{c}\right)+\beta T\left(e_{2} e_{1}^{c}\right) \\
& =\alpha T\left(e_{1} e_{2}\right)+\alpha T\left(e_{1} e_{2}^{c}\right)+\beta T\left(e_{1} e_{2}\right)+\beta T\left(e_{2} e_{1}^{c}\right) \\
& =\alpha T\left(e_{1} e_{2}\right) \vee \alpha T\left(e_{1} e_{2}^{c}\right)+\beta T\left(e_{1} e_{2}\right) \vee \beta T\left(e_{2} e_{1}^{c}\right) \\
& =\alpha T\left(\left(e_{1} e_{2}\right) \vee\left(e_{1} e_{2}^{c}\right)\right)+\beta T\left(\left(e_{1} e_{2}\right) \vee\left(e_{2} e_{1}^{c}\right)\right) \\
& =\alpha T\left(e_{1}\right)+\beta T\left(e_{2}\right) .
\end{aligned}
$$

A simple combination between the two previous steps gives the desired result.

The following proposition is a surprising and interesting consequence of the above result.

PROposition 2. Let A be a Dedekind complete vector lattice with a strong order unit $e$ and let $B$ be a vector lattice. If $T$ is a lattice homomorphism from $A$ into $B$ such that $T(\lambda e)=\lambda T(e)$ for each $\lambda \in \mathbb{R}_{+}$, then $T$ satisfies the following properties:

(1) $T(a+b)=T(a)+T(b)$ for each $a, b \in A_{+}$;

(2) $T(\lambda a)=\lambda T(a)$ for each $\lambda \in \mathbb{R}_{+}$.

Proof. Let $a, b \in A_{+}$, let $\lambda \in \mathbb{R}_{+}$and let

$$
L=\left\{k_{n} \in A ; k_{n}=\sum_{i=1}^{i=n} \alpha_{i} e_{i}, \alpha_{i} \in \mathbb{R}_{+}, e_{i} \text { is a component of } e, n=1,2, \ldots\right\} .
$$

By using the Freudenthal spectral theorem [5, Theorem 40.2], there exist two sequences $\left(k_{n}\right)_{n},\left(l_{n}\right)_{n}$ such that $k_{n}, l_{n} \in L, k_{n} \nearrow a($ r.u. $)$ and $l_{n} \nearrow b($ r.u. $)$. It follows that there exists $n_{0} \in \mathbb{N}^{*}$ such that, for all $n \geq n_{0}$, we have

$$
0 \leq a-k_{n} \leq e / n \text { and } 0 \leq b-l_{n} \leq e / n .
$$

It follows that

$$
0 \leq a \leq(e / n)+k_{n} \quad \text { and } \quad 0 \leq b \leq(e / n)+l_{n}
$$

for all $n \geq n_{0}$. Then

$$
0 \leq a+b \leq 2(e / n)+k_{n}+l_{n} .
$$

By applying the lattice homomorphism $T$, we deduce that

$$
0 \leq T(a) \leq T\left((e / n)+k_{n}\right) \quad 0 \leq T(\lambda a) \leq T\left(\lambda(e / n)+\lambda k_{n}\right)
$$


and

$$
0 \leq T(b) \leq T\left((e / n)+l_{n}\right) .
$$

By using the previous proposition,

$$
\begin{gathered}
T\left((e / n)+k_{n}\right)=T(e / n)+T\left(k_{n}\right)=(1 / n) T(e)+T\left(k_{n}\right) \\
T\left(\lambda(e / n)+\lambda k_{n}\right)=T(\lambda(e / n))+T\left(\lambda k_{n}\right)=\lambda(1 / n) T(e)+\lambda T\left(k_{n}\right)
\end{gathered}
$$

and

$$
T\left((e / n)+l_{n}\right)=T(e / n)+T\left(l_{n}\right)=(1 / n) T(e)+T\left(l_{n}\right) .
$$

Therefore

$$
T\left(k_{n}\right) \nearrow T(a)(\text { r.u. }), T\left(\lambda k_{n}\right) \nearrow T(\lambda a)(\text { r.u. }) \quad \text { and } T\left(l_{n}\right) \nearrow T(b)(\text { r.u. }) .
$$

Again, by the previous proposition, we have $T\left(\lambda k_{n}\right)=\lambda T\left(k_{n}\right)$. Then

$$
T(\lambda a)=\lambda T(a) .
$$

Moreover, by the previous proposition, we obtain $T\left(k_{n}\right)+T\left(l_{n}\right)=T\left(k_{n}+l_{n}\right)$. Since $T\left(k_{n}\right)+T\left(l_{n}\right) \nearrow T(a)+T(b)(r . u$.$) and since k_{n}+l_{n} \nearrow a+b($ r.u. $)$ and by applying the map $T$ to the inequality (3.5), we find that $T\left(k_{n}+l_{n}\right) \nearrow T(a+b)(r . u$.$) .$ Then $T(a)+T(b)=T(a+b)$ and we are done.

The following is essential to prove the first main result.

Proposition 3. Let $A$ be a vector lattice, let $A^{\delta}$ be its Dedekind completion and let $B$ be a Dedekind complete vector lattice. If $T$ is a lattice homomorphism from A into $B$, then $T_{/ A_{+}}$has an extension to a lattice homomorphism of $\left(A^{\delta}\right)_{+}$into $B$.

PRoOf. Let $x \in\left(A^{\delta}\right)_{+} \backslash A_{+}$and let $M=\left\{(x \vee a) \wedge b, a, b \in A_{+}\right\}$. It is clear that $M$ is the sublattice of $\left(A^{\delta}\right)_{+}$generated by $x$ and $A_{+}$. Let us define

$$
T_{1}(x)=\sup \left\{T(z), z \leq x, z \in A_{+}\right\} .
$$

Thus, we can define $T^{\prime}: M \rightarrow B$ by

$$
T^{\prime}((x \vee a) \wedge b)=\left(T_{1}(x) \vee T(a)\right) \wedge T(b) \quad\left(a, b \in A_{+}\right) .
$$

Clearly $T^{\prime}$ is a lattice homomorphism of $M$ into $B$. By Zorn's lemma, $T^{\prime}$ has a maximal extension to a lattice homomorphism $T^{*}$ of a sublattice $N$ of $\left(A^{\delta}\right)_{+}$ into $B$. We prove that $N=\left(A^{\delta}\right)_{+}$. Suppose that there exists $y \in\left(A^{\delta}\right)_{+} \backslash N$. Let $P=\{(y \vee a) \wedge b, a, b \in N\}$. Then $P$ is the sublattice of $\left(A^{\delta}\right)_{+}$generated by $y$ and $N$. Let us define

$$
T_{1}^{*}(y)=\sup \{T(z), z \leq y, z \in N\} .
$$

Then we can define $T^{\sharp}: P \rightarrow B$ by

$$
T^{\sharp}((y \vee a) \wedge b)=\left(T_{1}^{*}(y) \vee T^{*}(a)\right) \wedge T^{*}(b) \quad(a, b \in N) .
$$

Therefore, $T^{\sharp}$ is a lattice homomorphism of $P$ into $B$. This contradicts maximality of $T^{*}$ and so $N=\left(A^{\delta}\right)_{+}$, as required. 
We are now in position to give a generalized version of the Mena-Roth theorem for lattice homomorphisms on vector lattices with strong order units. The proof is identical in concept to [3, Lemma 1].

THEOREM 4. Let $A$ be a vector lattice with a strong order unit e and let $B$ be a vector lattice. If $T$ is a lattice homomorphism from $A$ into $B$ such that $T(\lambda e)=\lambda T(e)$ for each $\lambda \in \mathbb{R}_{+}$, then $T$ is linear.

PROOF. It is shown in the previous proposition that $T_{/ A_{+}}$has an extension to a lattice homomorphism of $\left(A^{\delta}\right)_{+}$into the vector lattice Dedekind completion $B^{\delta}$ of $B$. According to Proposition 2, $T$ is additive from $\left(A^{\delta}\right)_{+}$into the Dedekind completion of $B$ and so on $A_{+}$. It is well known, by [1, Theorem 1.7] that $T$ extends uniquely to a positive operator $T^{\prime}$ from $A$ to $B$. Hence,

$$
T^{\prime}(x)=T\left(x^{+}\right)-T\left(x^{-}\right) .
$$

Since $T$ is a lattice homomorphism,

$$
(T(x))^{-}=(-T(x)) \vee 0=-(T(x) \wedge 0)=-T(x \wedge 0)=-T\left(-x^{-}\right)=T\left(x^{-}\right) .
$$

Hence,

$$
T^{\prime}(x)=T\left(x^{+}\right)-T\left(x^{-}\right)=(T(x))^{+}-(T(x))^{-}=T(x) .
$$

Therefore, $T=T^{\prime}$.

The next corollary improves earlier results of [3, 4, 6] and [8] which assumed uniform completeness.

Corollary 5. Let $A$ be a vector lattice with a strong order unit e and let $B$ be a vector lattice. If $T$ is a lattice homomorphism from $A$ into $B$ such that $T(\lambda e)=\lambda T(e)$ for each $\lambda \in \mathbb{R}$, then $T$ is linear.

The following results are a consequence of the above theorem.

COROllary 6. Let $A$ be a vector lattice with a weak order unit e and let $B$ be a vector lattice with a weak order unit $f$. If $T$ is a lattice homomorphism from $A$ into $B$ such that $T(\lambda e)=\lambda f$ for each $\lambda \in \mathbb{R}_{+}$, then $T$ is linear.

Proof. Let $a, b \in A$ and let $g=|a|+|b|$ and let $\beta \in \mathbb{R}_{+}$. According to the previous theorem, $T$ is linear on $A_{e}$ and since $g \wedge n e, e \in A_{e}$,

$$
\begin{aligned}
T(\beta g) & =\sup (T(\beta g) \wedge n f) \\
& =\sup (T(\beta g) \wedge \beta n f) \\
& =\sup (T(\beta g) \wedge \beta T(n e)) \\
& =\sup (T(\beta g \wedge \beta n e)) \\
& =\beta \sup (T(g \wedge n e))
\end{aligned}
$$


for each $\beta \in \mathbb{R}_{+}$. Moreover,

$$
\begin{aligned}
\beta T(g) & =\sup (\beta T(g) \wedge n f) \\
& =\sup (\beta T(g) \wedge \beta n f) \\
& =\sup (\beta T(g) \wedge \beta T(n e)) \\
& =\beta \sup (T(g) \wedge T(n e)) \\
& =\beta \sup (T(g \wedge n e))
\end{aligned}
$$

for each $\beta \in \mathbb{R}_{+}$. Therefore, $T(\beta g)=\beta T(g)$, for each $\beta \in \mathbb{R}_{+}$. Again by the fact that $T$ is linear on $A_{e}$, we have

$$
\begin{aligned}
\beta T(g)+\beta T(e) & =T(\beta g)+T(\beta e) \\
& =\sup (T(\beta g \wedge n e))+T(\beta e) \\
& =\sup (T(\beta g \wedge n e)+T(\beta e)) \\
& =\sup (T(\beta g \wedge n e+\beta e)) \\
& =\sup (T((\beta g+\beta e) \wedge(n+\beta) e)) \\
& =\sup (T(\beta g+\beta e) \wedge(n+\beta) f) \\
& =\sup (T(\beta g+\beta e) \wedge n f) \\
& =T(\beta g+\beta e)
\end{aligned}
$$

for each $\beta \in \mathbb{R}_{+}$. Therefore, $T_{/ A_{g+e}}: A_{g+e} \rightarrow B$ is a lattice homomorphism which satisfies $T(\beta g+\beta e)=T(\beta g)+T(\beta e)=\beta T(g)+T(\beta e)$ for each $\beta \in \mathbb{R}_{+}$. According to the previous results, $T_{/ A_{g+e}}$ is linear. Since $a+\lambda b \in A_{g+e}$, for each $\lambda \in \mathbb{R}$, it follows that $T(a+\lambda b)=T(a)+\lambda T(b)$, for each $\lambda \in \mathbb{R}$, which gives the desired result.

As a consequence, we deduce a result of Ercan and Wickstead [3, Lemma 1].

COROLLARY 7. Let A be a uniformly complete vector lattice with a weak order unite and let $B$ be a vector lattice with a weak order unit $f$. If $T$ is a lattice homomorphism from $A$ into $B$ such that $T(\lambda e)=\lambda f$ for each $\lambda \in \mathbb{R}$, then $T$ is linear.

Next, we broach the problem of finding a sufficient condition for a lattice homomorphism of vector lattices, which do not have a weak order unit, to be linear. This will culminate in a second version of the Mena-Roth theorem and the Ercan-Wickstead theorem.

THEOREM 8. Let $A, B$ be vector lattices and let $\left(u_{i}\right)_{i \in I},\left(v_{i}\right)_{i \in I}$ be maximal orthogonal systems of $A$ and $B$, respectively. If $T$ is a lattice homomorphism from $A$ into $B$ such that $T\left(\lambda u_{i}\right)=\lambda v_{i}$ for each $\lambda \in \mathbb{R}_{+}$and $i \in I$, then $T$ is linear. 
Proof. It is shown in Proposition 3, that $T_{/ A_{+}}$has an extension to a lattice homomorphism of $\left(A^{\delta}\right)_{+}$into the vector lattice Dedekind completion $B^{\delta}$ of $B$. According to Proposition 2, $T$ is additive on $\left(A^{\delta}\right)_{+}$. Let $x, y \in A_{+}$and $\lambda \in \mathbb{R}_{+}$. Then

$$
\begin{aligned}
T(x) & =\sup _{H, n}\left(\sum_{i \in H}\left(T(x) \wedge n T\left(u_{i}\right)\right)\right) \\
& =\sup _{H, n}\left(\sum_{i \in H} T\left(x \wedge n u_{i}\right)\right) \\
& =\sup _{H}\left(\sum_{i \in H} T\left(x_{i}\right)\right)
\end{aligned}
$$

where $x_{i}$ is the projection component of $x$ in the order band generated $u_{i}$ in $A^{\delta}$ (denoted by $B_{u_{i}}$ ) and $H$ is a finite subset of $I$. Hence,

$$
T(\lambda x)=\sup _{H}\left(\sum_{i \in H} T\left(\lambda x_{i}\right)\right) .
$$

By Corollary $6, T_{/ B_{u_{i}}}$ is linear, then $T\left(\lambda x_{i}\right)=\lambda T\left(x_{i}\right)$. Hence, $T(\lambda x)=\lambda T(x)$. Moreover,

$$
\begin{aligned}
T(x+y) & =\sup _{H}\left(\sum_{i \in H} T\left((x+y)_{i}\right)\right) \\
& =\sup _{H}\left(\sum_{i \in H} T\left(x_{i}+y_{i}\right)\right) \\
& =\sup _{H}\left(\sum_{i \in H}\left(T\left(x_{i}\right)+T\left(y_{i}\right)\right)\right) \\
& =\sup _{H}\left(\sum_{i \in H} T\left(x_{i}\right)\right)+\sup _{H}\left(\sum_{i \in H} T\left(y_{i}\right)\right) \\
& =T(x)+T(y) .
\end{aligned}
$$

To complete the proof, it is sufficient to use [1, Theorem 1.7], as in the previous theorem.

We finish this paper with the following remark.

REMARK 9. We note that, in the results of Mena and Roth [6], Thanh [8], Lochan and Strauss [4] and Ercan and Wickstead [3], the assumption that $A$ is a uniformly complete vector lattice is superfluous, as shown in the previous results.

\section{Acknowledgement}

I am grateful to the anonymous referee for pointing out an error in my previous version and for providing suggestions that greatly improved the paper. 


\section{References}

[1] C. D. Aliprantis and O. Burkinshaw, Positive Operators (Academic Press, Orlando, FL, 1985).

[2] B. De Pagter, $f$-Algebras and orthomorphisms. Thesis, Leiden, 1981.

[3] Z. Ercan and A. W. Wickstead, 'When a lattice homomorphism is a Riesz homomorphism', Math. Nachr. 279(9-10) (2006), 1024-1027.

[4] R. Lochan and D. Strauss, 'Lattice homomorphisms of spaces of continuous functions', J. London Math. Soc. (2) 25 (1982), 379-384.

[5] W. A. J. Luxemburg and A. C. Zaanen, Riesz Spaces I (North-Holland, Amsterdam, 1971).

[6] R. Mena and B. Roth, 'Homomorphisms of lattices on continuous functions', Proc. Amer. Math. Soc. 71 (1978), 11-12.

[7] H. H. Schaefer, Banach Lattices and Positive Operators, Grundlehren Math. Wiss., 215 (Springer, Berlin, 1974).

[8] D. T. Thanh, 'A generalization of a theorem of R. Mena and B. Roth', Ann. Univ. Sci. Budapest. Eotvos Sect. Math. 34 (1992), 167-171.

MOHAMED ALI TOUMI, Département de Mathématiques,

Faculté des Sciences de Bizerte, 7021, Zarzouna, Bizerte, Tunisia

e-mail: mohamedali.toumi@fsb.rnu.tn 